\title{
An Analytical Model for the Contention Access Period of the Slotted IEEE 802.15.4 with Service Differentiation
}

\author{
Eugène David Ngangue Ndih* Nadia Khaled*† Giovanni De Micheli* \\ * School of Computer and Communications Sciences \\ Swiss Federal Institute of Technology in Lausanne, Switzerland \\ Email: \{eugenedavid.nganguendih,nadia.khaled,giovanni.demicheli\}@epfl.ch \\ $\dagger$ Department of Signal Theory and Communications \\ University of Carlos III, Madrid, Spain
}

\begin{abstract}
The IEEE 802.15.4 standard is poised to become the global standard for low data rate, low energy consumption wireless sensor networks (WSN). By assigning the same sets of contention access parameters for all data frames and nodes, the contention access period (CAP) of the slotted IEEE 802.15.4 medium access control (MAC) currently provides a priorityindependent channel access functionality and no service differentiation. Several recent WSN applications such as wireless body sensor networks, however, may require service differentiation and traffic prioritization support to accommodate potential highpriority traffic (e.g., alarms or emergency alerts). By allowing different sets of access parameters and data frame lengths for different priority classes, this paper develops a Markov-chain-based analytical model of the CAP of the IEEE 802.15.4 MAC with service differentiation, under unsaturated traffic conditions. In particular, given two priority classes, our analytical model is used to evaluate the performance of a simple, yet effective, contentionwindow-based service differentiation strategy, in terms of the resulting throughput, average frame service time and access priority for each priority class. The accuracy of the analytical model is validated by extensive $n s-2$ simulation.
\end{abstract}

\section{INTRODUCTION}

The IEEE 802.15.4 standard is poised to become the global standard for low data rate, low energy consumption wireless sensor networks (WSN) for a wealth of application areas, such as environment monitoring, industrial process surveillance, home automation and personal health monitoring. Since its ratification, the IEEE 802.15.4 MAC has received much interest to assess its throughput and energy performance. In particular, the novel slotted access protocol featured in the contention access period (CAP) of its beacon-enabled mode has spurred much attention; many preliminary simulation studies [1], [2] were conducted, and several accurate analytical models have been introduced. These analytical models follow two main approaches. The first approach is inspired from the seminal work of Bianchi on the analysis of IEEE 802.11 [3], and is based on modelling the behavior of a sensing node using a Markov chain. The most relevant contributions along this approach are [4], which adapts Bianchi's model to the specific contention access procedure of the slotted IEEE 802.15.4 CAP under unacknowledged saturated traffic conditions, [5] which corrects the analysis in [4] and extends it to include acknowledgment transmission, and [6] which proposes a more complex model reflecting additionally the superframe structure under unsaturated traffic conditions. A final relevant contribution, upon which our work is based, is the work of Ramachandran et al. [7] which develops a framework based on two Markov chains; the node-state chain to determine the fraction of time a node spends in different states and the channel-state chain to calculate the throughput of the network. Our choice is motivated by the demonstrated accuracy of this model with respect to ns-2 simulations, as well as its simplicity and amenability for extension. The second approach to analytically model the IEEE 802.15.4 CAP alternatively models the contention access mechanism as an embedded Markov renewal process and performs a fixed-point analysis to calculate the throughput of the network [8].

By assigning the same sets of contention access parameters for all data frames and nodes, the CAP of the slotted IEEE 802.15.4 MAC currently provides a priority-independent channel access functionality and no service differentiation. However, several wireless sensor network applications may require service differentiation and traffic prioritization support to accommodate delay sensitive traffic (e.g., alarms or emergency alerts in wireless body sensor networks) or to prioritize high relevance/reliability messages throughout a sensor network. This work seeks such service differentiation provision in the slotted IEEE 802.15.4 CAP through defining different priority classes, each with its specific values of access parameters and data frame length, following the example of the IEEE 802.11e quality-of-service $(\mathrm{QoS})$-aware extension of the original IEEE 802.11 wireless local area networks MAC. More specifically, this paper extends the Markov-chain-based analytical model of the IEEE 802.15.4 CAP in [7] in the presence of two priority classes (high and low). To the best of our knowledge, no such model exists for the IEEE 802.15.4 CAP with service differentiation. As a matter of fact, the only tentative model of differentiated access in the IEEE 802.15.4 MAC was presented in [9], and is not mathematically sound as it incorrectly assumes that the packet sending probability is independent 
from node to node, instead of the channel sensing probability. Finally, a similar accurate model does exist for the IEEE 802.11e enhanced distributed coordination function [10], but is not directly applicable to the IEEE 802.15.4 CAP due to their different contention access procedures.

The rest of the paper is organized as follows. Section II reviews the slotted IEEE 802.15.4 MAC protocol, and the underlying slotted CSMA/CA algorithm. Section III introduces our extension of the state-of-the-art IEEE 802.15.4 analytical model to include service differentiation. This extended analytical model is validated and used to evaluate the performance of a contention-window-based service differentiation strategy in Section IV. Finally, the paper is concluded in Section V.

\section{OVERVIEW OF THE SLOtTEd IEEE 802.15.4 CSMA/CA Channel ACCess Algorithm}

In this section, we briefly review the MAC protocol of the slotted mode of the IEEE 802.15.4 standard, which specifies the time structure, default parameter settings and channel access procedure for beacon-enabled networks. A comprehensive description of the standard may be found in [11], [1]

In the slotted mode, a coordinator periodically transmits a beacon to identify its network, synchronize the nodes associated with it, and delimit the superframe time structure that organizes communication in the network. The latter superframe begins with a beacon, followed by an active and an optional inactive period. All communication takes place during the active period, whereas all nodes may power down to conserve energy during the inactive period. The active period of a superframe further consists of a contention access period (CAP) and an optional contention-free period (CFP). In the CFP, the coordinator controls the channel access by assigning guaranteed time slots (GTS) to those nodes which request them. Alternatively, the CAP allows nodes to access the channel in a distributed fashion through a slotted CSMA/CA algorithm, which is specified in the standard as follows.

In the slotted CAP, all nodes are synchronized and can begin transmission only at the boundaries of time limits called backoff slots. The duration of one backoff slot is aUnitBackof $f$ Period (default value $=3.2 \mathrm{~ms}$ ). When a node has a new data frame waiting for transmission at the MAC buffer, it first initializes the three relevant contention parameters, namely the number of random backoff stages experienced $(N B)$ to 0 , the current backoff exponent $(B E)$ to macMinBE (default value $=3$ ) and the contention window $(C W)$ (default value $=2)$. Then, it selects a backoff counter value uniformly from the window $\left[0,2^{B E}-1\right]$. This backoff counter value is decremented by one for each backoff slot regardless of the channel state. When the backoff counter reaches zero, the node performs carrier sensing that consists of clear channel assessment (CCA) for the next $C W$ consecutive backoff slots. If the channel is sensed idle during the first CCA, $C W$ is decremented by one and the node performs the following CCA at the next backoff slot boundary. Only when the channel is assessed idle during the $C W$ consecutive CCAs, will the node start transmission in the next backoff slot. Otherwise, the node will enter the next backoff stage; it will increase the values of $N B$ and $B E$ by one, reset $C W$ to its initial value and draw a new random number of backoff slots from the updated window $\left[0,2^{B E}-1\right]$ to wait before the channel may be sensed again. This procedure is repeated until the frame is transmitted, or a channel access failure is declared. The latter occurs when $N B$ reaches a maximum number of macMaxCSMABackoffs allowed random backoff stages (default value=5). Note that $B E$ shall not be incremented beyond its maximum value $a M a x B E$ (default value=5); after this value, $\mathrm{BE}$ is frozen to $a M a x B E$.

\section{Analytical Model}

This section extends the analytical model of the IEEE 802.15.4 CAP in [7] in the presence of two priority classes: high-priority and low-priority nodes. As such, the extended model analogously develops two node-state chains corresponding to the two classes of nodes, and a channel-state chain. Although this analytical model is able to capture more general service differentiation strategies (based on different macMinBE, aMaxBE, $C W$ ), the present paper focuses on the $C W$-based service differentiation strategy consisting in allowing the high-priority nodes to use $C W=1$ (class 1), instead of the default $C W=2$ used by the lowpriority transmissions (class 2). Maintaining the same backoff procedure parameters, this strategy reduces the duration of idle channel sensing for high-priority nodes so that they gain access to the channel ahead of low-priority nodes, which must observe a longer channel idle time before transmission. All other contention access parameters are set to the default values previously indicated in Section II. We consider a single-hop wireless sensor network consisting of a coordinator, $M_{1}$ class1 nodes and $M_{2}$ class- 2 nodes. Furthermore, we consider a star topology and uplink data transmission scenario without acknowledgment.

\section{A. Model Assumptions and Approximations}

As an extension of the analytical model introduced in [7], the present work is based on the same assumptions and approximations which accuracy have been validated therein. For self-containment, the most salient ones are herein summarized: (1) All nodes are perfectly time-synchronized with the coordinator's beacon and are within the transmission range of each other (no hidden-node problem); (2) The nodes are not allowed to sleep and the entire superframe duration is active and set to be large $(B O=S O=6)[11]$ in order to accurately approximate the CSMA/CA scheme as non-persistent with backoff and neglect the effect of the finite superframe [2]; (3) Once assigned to a priority class, a node remains in the same class for the whole analysis; (4) All of the active period of the superframe is allocated to the CAP, as it is the focus of this work; (5) Only direct transmission is considered and the coordinator is configured not to acknowledge the reception of the packets which have a fixed duration of $N_{1}$ and $N_{2}$ backoff slots, for class- 1 and class- 2 nodes respectively; (6) Initially, the nodes have no packet to transmit and receive 
packets for transmission according to a Poisson arrival rate of $\lambda_{1}$ and $\lambda_{2}$ packets per packet duration, for class-1 and class-2 nodes respectively. Moreover, the unsaturated traffic assumption entails that no packet buffering is allowed while a node is transmitting or has already initiated a CSMA/CA process; (7) The uniform distribution specified in the IEEE 802.15.4 standard, to draw the number of backoff slots a node has to wait at each backoff stage of the contention access procedure, is replaced with a geometric distribution of the same mean number of backoff slots so that the backoff procedure remains memoryless. Note that the two classes of nodes use the same default backoff parameters and distributions and are only differentiated by their clear channel assessment procedure (i.e., $C W$-based channel access differentiation).

Notation: All probabilities associated with channel states have a superscript ' $c$ ' (e.g., $p_{i}^{c}$ ), and those associated with node states have a superscript ' $n$ ' coupled to a superscript $q \in\{1,2\}$ indicating the class of the node (e.g., $p_{k}^{n, q}$ for a class-q node).

\section{B. Nodes State Models}

The behavior of each class of node is modeled by means of a corresponding discrete-time Markov chain: Figure 1 depicts that of an individual class-1 node, while Figure 2 illustrates the Markov chain of a class- 2 node. Initially, a class-q node is in

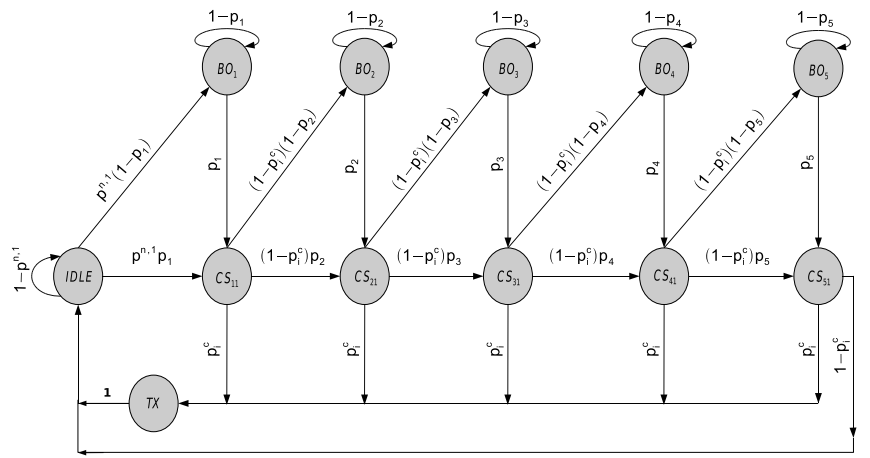

Fig. 1. Embedded Markov chain model for a class-1 sensing node

the IDLE state and would remain in that state, until it receives a packet for transmission with probability $p^{n, q}=\frac{\lambda_{q}}{N_{q}}$, since class-q nodes receive packets of $N_{q}$ backoff slots of duration arriving with a Poisson arrival rate of $\lambda_{q}$ packets per packet duration. Once the class-q node has a packet to transmit, it would spend in backoff state $B O_{1}$ a random number of backoff slots $X_{1}$ geometrically distributed according to $P\left[X_{1}=k\right]=$ $\left(1-p_{1}\right)^{k} p_{1}$ for $k=0,1, \cdots, \infty$, where the parameter $p_{1}$ is set to $1 / 4.5$, so that the corresponding random distribution has the same mean number of backoff slots as its counterpart IEEE 802.15.4 uniform backoff distribution, i.e., 3.5 [7].

Upon leaving $B O_{1}$, the node moves to state $C S_{11}$, which corresponds to performing the first CCA. If the channel is found idle in the first CCA, which occurs with probability $p_{i}^{c}$, a class-1 node would enter the transmit state $T X$ and start transmitting its packet during $N_{1}$ backoff slots. Alternatively, a class-2 node would have to move into state $C S_{12}$ and

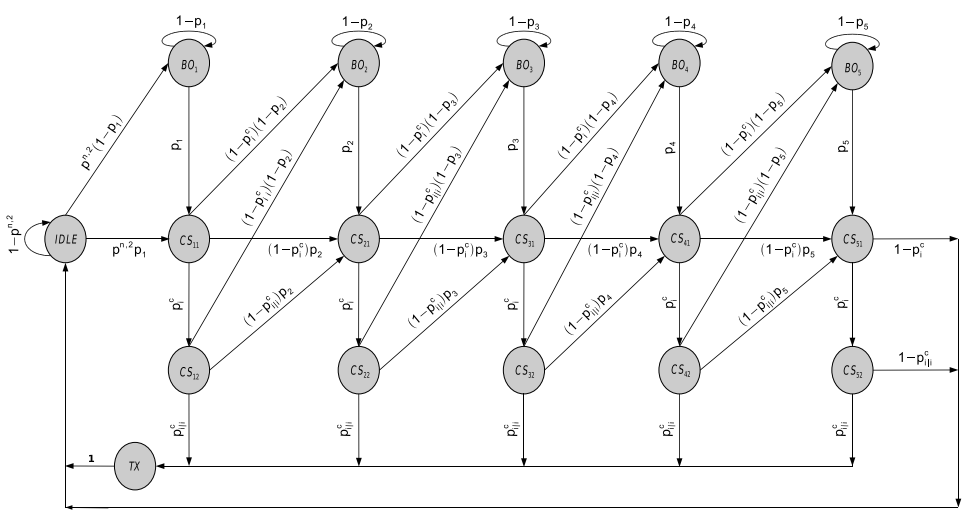

Fig. 2. Embedded Markov chain model for a class-2 sensing node

perform the second CCA. If the channel is again found idle for the second consecutive time, an event characterized by the conditional probability $p_{i \mid i}^{c}$, the class- 2 node would then enter the transmit state $T X$ and spend therein $N_{2}$ backoff slots. Once packet transmission is completed, both nodes transition to the IDLE state with probability 1 . If the channel is found busy when a class-1 node is in state $C S_{11}$ (resp. when a class-2 node is in states $C S_{11}$ or $C S_{12}$ ), which happens with probability $\left(1-p_{i}^{c}\right)$ (resp. with probabilities $\left(1-p_{i}^{c}\right)$ and $\left.\left(1-p_{i \mid i}^{c}\right)\right)$, then the node transitions into the second backoff stage $\mathrm{BO}_{2}$ and repeats the same backoff and clear channel assessment procedure to the first stage, where the backoff parameter $p_{1}$ is updated to $p_{2}=1 / 8.5$ to reflect the fact that $B E=4$ for $\mathrm{BO}_{2}$. The number of backoff slots $\mathrm{X}_{2}$ the node spends in $\mathrm{BO}_{2}$ is again geometrically distributed: $P\left[X_{2}=k\right]=\left(1-p_{2}\right)^{k} p_{2}$. In general, we adopt the notation $B O_{1 \leq i \leq 5}$ to represent the 5 random backoff stages and $C S_{i j}: 1 \leq i \leq 5,1 \leq j \leq q$ to denote the $j^{t h}$ carrier sense attempt after the $i^{t h}$ random backoff stage $B O_{i}$ of a class-q node. Accordingly, the complete Markov chains of Figures 1 and 2 are constructed starting from the IDLE state until either the packet has been successfully transmitted or the maximum number of allowed random backoff stages is reached and a channel access failure is declared. For completeness, note that $p_{3}=p_{4}=p_{5}=1 / 16.5$ since $B E=5$ for $B O_{3 \leq i \leq 5}$ [7].

Let $p_{t}^{n, 1}$ and $p_{t}^{n, 2}$ denote the probability that a class- 1 and a class-2 node, respectively, begins transmission in a generic backoff slot. According to Figures 1 and 2, a node will begin a transmission in the next backoff slot if, being in the last CS state of any backoff stage, it senses the channel idle. Noting that the probability of being in the last CS state of any backoff stage is simply the long-term proportion of time the chain spends in $\cup_{j=1}^{2} \cup_{i=1}^{5} C S_{i j}, p_{t}^{n, 1}$ and $p_{t}^{n, 2}$ are given by:

$$
\begin{aligned}
p_{t}^{n, 1} & =\frac{\sum_{i=1}^{5} \pi_{c s_{i 1}}^{n, 1}}{1+\left(N_{1}-1\right) \pi_{t x}^{n, 1}} p_{i}^{c} \\
p_{t}^{n, 2} & =\frac{\sum_{i=1}^{5} \pi_{c s_{i 2}}^{n, 2}}{1+\left(N_{2}-1\right) \pi_{t x}^{n, 2}} p_{i \mid i}^{c}
\end{aligned}
$$

where $\pi_{c s_{i 1}}^{n, 1}$ and $\pi_{t x}^{n, 1}$ are respectively the long-run proportion of transitions into states $C S_{i 1}$ and $T X$ of a class-1 node, 
while $\pi_{c s_{i 2}}^{n, 2}$ and $\pi_{t x}^{n, 2}$ correspond to the long-run proportion of transitions into states $C S_{i 2}$ and $T X$ of a class-2 node. Note that the long-run proportion of transitions into the different states of a class-q node can be obtained by solving its Markov chain, as detailed in [7]. Equations (1) and (2) are the two first independent equations of the system relating $p_{t}^{n, 1}, p_{t}^{n, 2}, p_{i}^{c}$ and $p_{i i}^{c}$ to be solved in order to determine the network behavior. The remaining two equations are subsequently obtained through solving the channel-state Markov chain.

\section{Channel State Model}

The channel behavior can be described using the discretetime Markov chain of Figure 3, which is constructed as follows. Given the maximum CCA span of 2 consecutive backoff

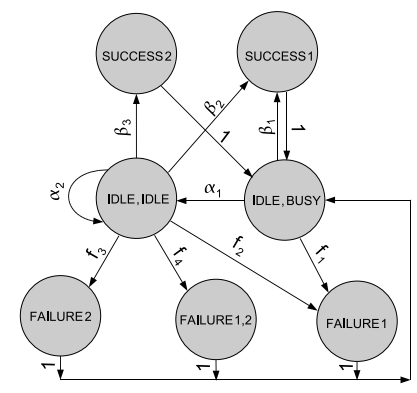

Fig. 3. Embedded Markov chain model for the channel

slots corresponding to $C W=2$ for class- 2 nodes, the event that the channel is idle in the current backoff slot encompasses two channel states: namely state (IDLE,IDLE) corresponding to the channel having been idle for two consecutive backoff slots, and state (IDLE,BUSY) capturing the case when the channel was busy during the previous backoff before becoming idle in the current backoff slot. Additionally to these two states, the channel may be in a success when a packet is successfully transmitted or a failure when a collision occurs. When the two priority classes have a common packet length $N$, these two events respectively correspond to a single SUCCESS channel state and a single FAILURE channel state, both of which have a dwell time of $N$ backoff slots. Our differentiated scenario, however, uses two different packet lengths for the two priority classes, and as such requires to further discriminate the channel's success and failure states in terms of the class or classes involved, as their dwell time differ. Therefore, our channelstate Markov chain discriminates the channel success state into: SUCCESS1, for the successful transmissions of class1 nodes, and SUCCESS2, for the successful transmissions of class-2 nodes. Similarly, the failure state is divided into three states depending on the class of the nodes and the length of the packets causing the state. Thus, state FAILURE1 corresponds to the collision of packets of class-1 nodes only. In state FAILURE2, the channel is experimenting the collision of packets transmitted by class-2 nodes exclusively. Finally, failure state (FAILURE1,2) is caused by colliding transmissions of class- 1 nodes and class- 2 nodes. The following describes the possible channel state transitions and their respective probabilities, according to Figure 3.
Let $p_{t \mid i}^{n, 1}$ be the conditional probability that a class- 1 node begins transmission given it has sensed the channel idle in the previous backoff slot, and $p_{t \mid i i}^{n, 2}$ be the conditional probability that a class- 2 node starts transmitting given it has sensed the channel idle during the two previous backoff slots:

$$
p_{t \mid i}^{n, 1}=\frac{p_{t}^{n, 1}}{p_{i}^{c}}, \quad p_{t \mid i i}^{n, 2}=\frac{p_{t}^{n, 2}}{p_{i i}^{c}}
$$

where $p_{i i}^{c}$ is the probability that the channel is idle in two consecutive backoff slots.

When the channel is in the (IDLE,BUSY) state, it may transition into one of 3 states: state (IDLE,IDLE) with probability $\alpha_{1}=\left(1-p_{t \mid i}^{n, 1}\right)^{M_{1}}$, which corresponds to the event that none of the $M_{1}$ class- 1 nodes begins the transmission; state SUCCESS 1 with probability $\beta_{1}=M_{1} p_{t \mid i}^{n, 1}\left(1-p_{t \mid i}^{n, 1}\right)^{M_{1}-1}$ corresponding to the event that a single class-1 node begins transmission in the current backoff slot; and state FAILURE1 with probability $f_{1}=\sum_{k=2}^{M_{1}}\left(\begin{array}{c}M_{1} \\ k\end{array}\right)\left(1-p_{t \mid i}^{n, 1}\right)^{M_{1}-k}\left(p_{t \mid i}^{n, 1}\right)^{k}$ that at least two class- 1 nodes simultaneously begin transmission in the current backoff slot.

When the channel is in the (IDLE,IDLE) state, it remains in that state if none of the nodes begins transmission, which occurs with probability $\alpha_{2}=\left(1-p_{t \mid i}^{n, 1}\right)^{M_{1}}\left(1-p_{t \mid i i}^{n, 2}\right)^{M_{2}}$. Otherwise, the channel will go into one of five states:

(1) State SUCCESS1: This transition occurs with probability $\beta_{2}=M_{1} p_{t \mid i}^{n, 1}\left(1-p_{t \mid i}^{n, 1}\right)^{M_{1}-1}\left(1-p_{t \mid i i}^{n, 2}\right)^{M_{2}}$, which corresponds to the event that only one class-1 node transmits while all remaining nodes abstain.

(2) State SUCCESS2: This happens with probability $\beta_{3}=$ $M_{2} p_{t \mid i i}^{n, 2}\left(1-p_{t \mid i i}^{n, 2}\right)^{M_{2}-1}\left(1-p_{t \mid i}^{n, 1}\right)^{M_{1}}$, when only one class-2 node begins transmission in the current backoff slot while all the other nodes do not.

(3) State FAILURE1: Representing the event that at least two of the $M_{1}$ class-1 nodes begin transmission and none of the class- 2 nodes does, this transition occurs with probability $f_{2}=\left(1-p_{t \mid i i}^{n, 2}\right)^{M_{2}} \sum_{k=2}^{M_{1}}\left(\begin{array}{c}M_{1} \\ k\end{array}\right)\left(p_{t \mid i}^{n, 1}\right)^{k}\left(1-p_{t \mid i}^{n, 1}\right)^{M_{1}-k}$.

(4) State FAILURE2: This event corresponds to at least two class- 2 nodes beginning transmission in the current backoff slot while none of the class-1 nodes does, and takes place with probability $f_{3}=\left(1-p_{t \mid i}^{n, 1}\right)^{M_{1}} \sum_{k=2}^{M_{2}}\left(\begin{array}{c}M_{2} \\ k\end{array}\right)\left(p_{t \mid i i}^{n, 2}\right)^{k}\left(1-p_{t \mid i i}^{n, 2}\right)^{M_{2}-k}$.

(5) State (FAILURE1,2): This corresponds to a collision between at least one class-1 node and at least one class- 2 node and happens with probability $f_{4}=1-\left(\alpha_{2}+\beta_{2}+\beta_{3}+f_{2}+f_{3}\right)$.

After being in a busy state, that is, either SUCCESS1, SUCCESS2, FAILURE1, FAILURE2 or (FAILURE1,2), the channel goes to the (IDLE,BUSY) state with probability 1.

The Markov chain of Figure 3 can be solved to determine the long-run proportions of transitions to states (IDLE,BUSY), (IDLE,IDLE), SUCCESS1, SUCCESS2, FAILURE1, FAILURE2 and (FAILURE1,2), denoted by $\pi_{i b}, \pi_{i i}, \pi_{s_{1}}, \pi_{s_{2}}, \pi_{f_{1}}$, 
$\pi_{f_{2}}$ and $\pi_{f_{12}}$ respectively. Accordingly, the steady-state probabilities, $p_{i b}^{c}$ and $p_{i i}^{c}$, that the channel is in the (IDLE,BUSY) and (IDLE,IDLE) states, respectively, are given by:

$$
p_{i b}^{c}=\frac{\pi_{i b}}{\sum_{k \epsilon \Omega} T_{k} \pi_{k}}, \quad p_{i i}^{c}=\frac{\pi_{i i}}{\sum_{k \in \Omega} T_{k} \pi_{k}}
$$

where $k, T_{k}$ denote channel state $k$ and its corresponding dwell time in backoff slots, $k$ belongs to the set of possible channel states $\Omega=\left\{i b, i i, s_{1}, s_{2}, f_{1}, f_{2}, f_{12}\right\}$. Given the packet length of each priority class, it is easily seen that $T_{i b}=T_{i i}=1, T_{s_{1}}=T_{f_{1}}=N_{1}$ and $T_{s_{2}}=T_{f_{2}}=N_{2}$, and $T_{f_{12}}=N_{m}=\max \left(N_{1}, N_{2}\right)$. Straightforward calculations lead to the following expression of the common denominator in (4):

$$
\begin{aligned}
& \sum_{k \in \Omega} T_{k} \pi_{k}=\pi_{i i}\left[1+\frac{\left(1-\alpha_{2}\right)\left(1+N_{1}\right)}{\alpha_{1}}\right. \\
& \left.+\left(N_{m}-N_{1}\right)\left(1-\alpha_{2}-\beta_{2}-f_{2}\right)-\left(N_{m}-N_{2}\right)\left(\beta_{3}+f_{3}\right)\right]
\end{aligned}
$$

Moreover, using the balance equation related to state (IDLE,IDLE), it is easily found that:

$$
\pi_{i b}=\frac{1-\alpha_{2}}{\alpha_{1}} \pi_{i i}
$$

$p_{i b}^{c}$ and $p_{i i}^{c}$ are readily computed through replacing (5) and (6) into the expressions in (4). The probability, $p_{i}^{c}$, that the channel is idle in a given backoff slot can then be derived, by noting that the channel could have been either idle or busy in the previous backoff slot, as follows: $p_{i}^{c}=p_{i i}^{c}+p_{i b}^{c}$. This equation gives $p_{i}^{c}$ as a function of $p_{t}^{n, 1}$ and $p_{t}^{n, 2}$, through the $p_{t \mid i}^{n, 1}$ and $p_{t \mid i i}^{n, 2}$ in the expressions of the transition probabilities of the channel-state chain. Together with $p_{i \mid i}^{c}=\frac{p_{i i}^{c}}{p_{i}^{c}}$, they provide the two missing independent equations that we couple with (1) and (2) in a consistent system of nonlinear equations, which can be solved numerically to completely resolve the node-state and channel-state chains.

\section{Metrics Formulation}

We are now ready to evaluate the three most relevant metrics capturing the service differentiation and traffic prioritization between the two priority classes.

1) Channel access probability: For each priority class, this probability corresponds to the probability that a node of this class begins transmission in a generic backoff slot. It is simply given by (1) and (2) for class 1 and class 2, respectively.

2) Aggregate channel throughput: The aggregate throughput of class $\mathrm{q}$ is defined as the fraction of time class- $\mathrm{q}$ nodes spend in the SUCCESSq state, i.e, it is the steady-state probability, $S_{q}$, of the channel being in the SUCCESSq state, derived from the channel Markov chain in Figure 3 as:

$$
S_{q}=\frac{N_{q} \pi_{s_{q}}}{\sum_{k \in \Omega} T_{k} \pi_{k}}
$$

where the denominator is given by (5), and the long-run proportion of transitions into channel states SUCCESS1, $\pi_{s_{1}}$, and SUCCESS2, $\pi_{s_{2}}$, are given by:

$$
\pi_{s_{1}}=\frac{\left(1-\alpha_{2}\right) \beta_{1}+\alpha_{1} \beta_{2}}{\alpha_{1}} \pi_{i i}, \quad \pi_{s_{2}}=\beta_{3} \pi_{i i}
$$

The respective aggregate throughputs of class 1 and class 2 directly follow from using the expressions of (8) and (5) into (7) for $q=1,2$.

3) Average frame service time: or MAC latency is defined as the average duration from the instant a packet becomes available for transmission to the end of its successful transmission. For class q, it is equal to the average duration between two successful class-q transmissions minus the average time spent by a class-q node in IDLE state during the latter duration:

$$
L_{q}=\frac{N_{q} M_{q}}{S_{q}}\left(1-p_{i}^{n, q}\right)
$$

where $p_{i}^{n, q}$ denotes the fraction of time the class-q node spends in the IDLE state, which was obtained from solving the class-q node state chain.

\section{Model Validation and Performance Results}

The simulations are performed using the 802.15.4 ns-2 module implemented by Zheng and Lee [1], their parameters are set according to III-A. We consider a star topology network with a coordinator and 12 sensing nodes equi-distributed among the two priority classes. In order to exclusively observe the effectiveness of CW-based differentiation as a service differentiation measure, we set the packet arrival rates $\lambda=$ $\lambda_{1}=\lambda_{2}$ and the packet length $N_{1}=N_{2}=10$ backoff slots. For this set-up, Figures 4, 5 and 6 illustrate the analytical and simulated channel access probabilities, aggregate throughput and latency of the two priority classes as a function of the packet arrival rate $\lambda$. They consistently show the excellent accuracy of our analytical model in capturing the various relevant performance metrics, and validate the various simplifying assumptions made in III-A according to [7]. Furthermore,

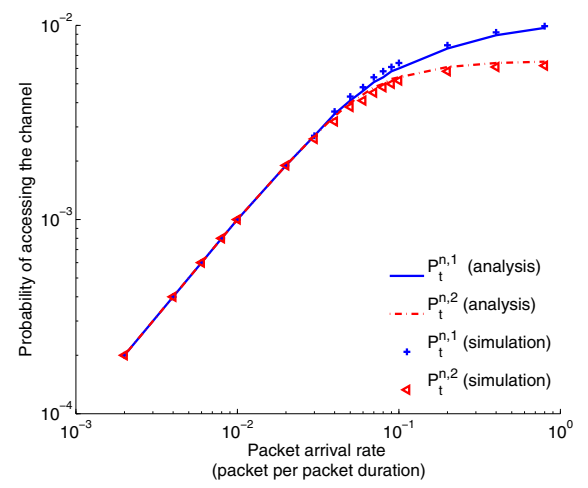

Fig. 4. Per-class channel access probability vs. the packet arrival rate $\lambda$

these performance plots reveal that, at low packet arrival rates, CW-based service differentiation does not have a noticeable effect on the throughput, the probability of accessing the medium or the latency since the nodes are mainly idle and have very low probability of attempting to access the channel at the same time; no service differentiation is needed. However, when the packet arrival rate increases beyond $\lambda=0.04$ packet per packet duration (corresponding to $10 \mathrm{kbps}$ since the channel capacity is $250 \mathrm{kbps}$ ), it appears clearly, in Figures 4, 5 


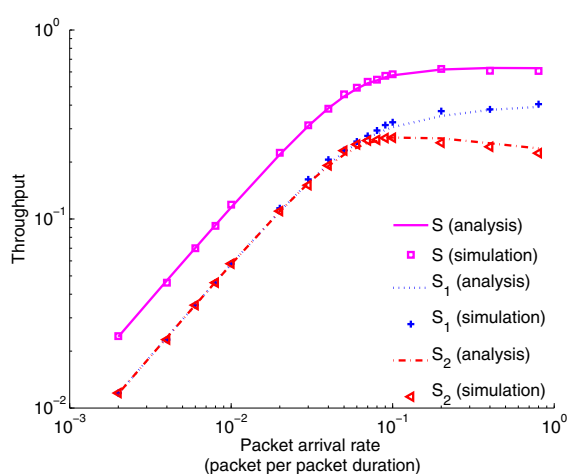

Fig. 5. Per-class and total aggregate throughput vs. packet arrival rate $\lambda$

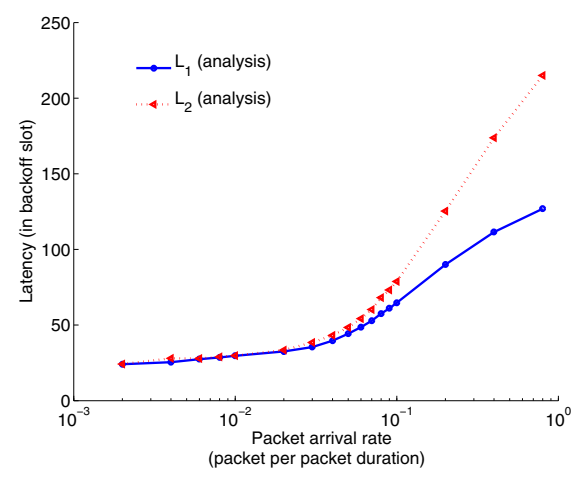

Fig. 6. Per-class latency vs. packet arrival rate $\lambda$

and 6 that class- 1 nodes benefit of a prioritized access to the channel, which translates into a larger throughput and up to a two-fold latency reduction at very high packet arrival rates. This effect is explained by the fact that when $\lambda$ increases, the number of packets available for transmission increases for both classes. Thus, the contention for the channel among the nodes increases, increasing the probability of two nodes of different classes starting their CCA procedure at the same time. In this specific case, the high-priority node gains access to the channel since it only has to perform a single CCA, and the low-priority node is deferred to the next backoff stage with the associated penalty of increasing its backoff exponent. This deference further disadvantages the class- 2 node with respect to other class-1 nodes, which may initiate a packet transmission with the initial backoff parameters. This explains the fact that class-1 nodes enjoy prioritized access to the channel and improved throughput, while class- 2 node suffer significantly larger latencies. Figure 5 also shows a decrease in the throughput of class- 2 nodes as $\lambda$ increases. It is due to increased collisions of their packets, which coupled to a saturation of their channel access probability (cf. Figure 4), induces a reduction in their throughput. This is not the case of class- 1 nodes, which channel access probability keeps growing and so does their throughput.

This service differentiation is maintained for different ratios of nodes between the two classes as shown in Figure 7, where we consider a fixed total number of sensing nodes $M=12$ and different node distributions: $M_{1}=M / 6$ (case 1 ), $M_{1}=M / 3$ (case 2), $M_{1}=M / 2$ (case 3), $M_{1}=2 M / 3$ (case 4) and $M_{1}=5 M / 6$ (case 5). Moreover, the larger $M_{1}$ is, the more disputed the channel access is by class- 1 nodes, which reduces the channel access probability of both classes.

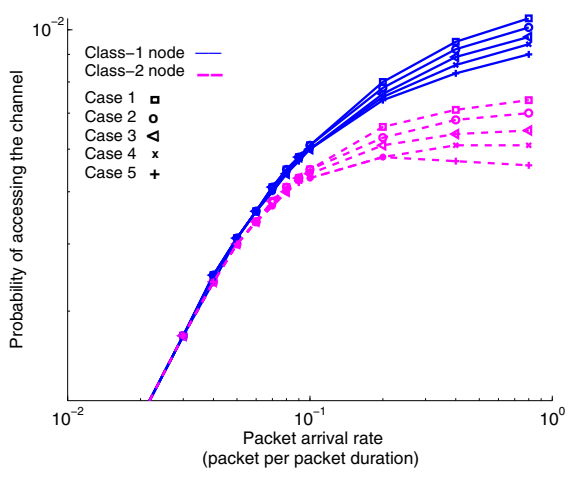

Fig. 7. Per-class channel access probability for different $\left(M_{1}, M_{2}\right)$ ratios

\section{CONCLUSION}

An analytical model was introduced to evaluate the effectiveness of the IEEE 802.15.4 CAP mechanisms and contention parameters to provide service differentiation and traffic prioritization. Its accuracy was illustrated in the evaluation of a simple, yet, effective $C W$-based service differentiation. We believe this analytical model to be instrumental in guiding the future appropriate configuration of these mechanisms and parameters for each priority class to achieve the service differentiation needs of single-hop WSNs applications.

\section{REFERENCES}

[1] J. Zheng and M. J. Lee, "Will IEEE 802.15.4 make ubiquitous networking reality? - a discussion on a potential low power, low bit rate standard," IEEE Comm. Mag., vol. 42, pp. 140-146, Jun. 2004.

[2] A. Koubaa, M. Alves, and E. Tovar, "A comprehensive simulation study of slotted CSMA/CA for IEEE 802.15.4 wireless sensor networks," in IEEE Proc. of Work. on Factory Comm. Sys., Jun. 2006, pp. 183-192.

[3] G. Bianchi, "Performance analysis of the IEEE 802.11 distributed coordination function," IEEE Jrl. on Selected Areas in Communications, vol. 18, no. 3, pp. 535-547, Mar. 2000.

[4] S. Pollin and et al., "Performance analysis of slotted carrier sense IEEE 802.15.4 MAC," in IEEE Proc. of GLOBECOM, Nov. 2006.

[5] R. K. Patro and et al., "Analysis and improvement of contention access protocol in IEEE 802.15.4 star network," in IEEE Proc. of Conf. on Mobile Adhoc and Sensor Systems (MASS), Oct. 2007, pp. 1-8.

[6] C. Y. Jung, H. Y. Hwang, D. K. Sung, and G. U. Hwang, "Enhanced markov chain model and throughut analysis of the slotted CSMA/CA for IEEE 802.15.4 under unsaturated traffic conditions," Accepted for future publication in IEEE Trans. on Vehicular Tech.

[7] I. Ramachandran, A. K. Das, and S. Roy, "Analysis of the contention access period of IEEE 802.15.4," ACM Trans. on Sensor Networks, vol. 3, no. 1, p. article 4, Mar. 2007.

[8] X. Ling and et al., "A renewal theory based analytical model for the contention access period of IEEE 802.15.4," IEEE Trans. on Wireless Comm., vol. 7, no. 6, pp. 2340-2349, Jun. 2008.

[9] E. Kim, M. Kim, S.-K. Youm, and C.-H. K. S. Choi, "Multi-level service differentiation scheme for the IEEE 802.15.4 networks," in Proc. of EUC Workshops, 2005, pp. 693-703.

[10] H. Zhu and I. Chlamtac, "Performance analysis for IEEE 802.11e EDCF service differentiation," IEEE Trans. on Wireles Comm., vol. 4, no. 4, pp. 1780-1788, Jul. 2005.

[11] "Wireless medium access control (MAC) and physical layer (PHY) specifications for low-rate wireless personal area networks (LR-WPANs)," IEEE, Tech. Rep. IEEE 802.15.4 Std., 2003. 